\title{
REAL TIME GENERIC OBJECT DETECTION AND TRACKING
}

\author{
Naman Swaroop \\ School Of Computer Science And \\ Engineering Galgotias University \\ Gautam Buddh Nagar, India
}

\begin{abstract}
This research paper, introduces a technique for automating the methodology of detecting and tracking objects using color feature and motion. Video Tracking is the process of finding a moving object over the priod of time using a camera. The main idea of video tracking is to track objects in consecutive frames of video. The relationship can give trouble when the objects are moving with speed with respect to the frame rate. A different situation may arise the uncertainity of the matter is the time when the object tracking changes orientation after eventually. For these circumstances video tracking frameworks typically utilize a movement model which depicts how the image of the target may change for distinctive conceivable movements of the object. Here in this paper we have developed an algorithm to to track object in video frames using color, motion and feature.
\end{abstract}

Key Terms-Color Feature, Motion Detection, Optical Flow.

\section{INTRODUCTION}

The advanced world is encased with colossal masses of computerized visual information. To investigate and comprehend this gigantic ocean of visual data, there exist many picture examination techniques. Those strategies that naturally perceive and identify the articles end up being of extraordinary utilize and give a noteworthy assistance in current applications and gadgets. The semantic and syntactic substance of the pictures and recordings can be perceived and further handled to get the essential information. The potential employments of the picture can be identified. The significant substance of picture is the items in the picture. There exists a noteworthy and fundamental requirement for object acknowledgment methods. Recognition is a significant undertaking in picture handling and $\mathrm{PC}$ vision. A lot of realized

\section{Motivation And Purpose}

- The fundamental inspiration driving this subject is that it is something that will exaggerate all the physical tasks. • Robotics and keen frameworks are humming around everywhere throughout the world. - Object acknowledgment and following diminishes human endeavors and gives efficiency.
- It is of enthusiasm as it might assist people with being mindful of moment data about specific articles and lessen human errands. - Automatic acknowledgment and extraction adds to the savvy frameworks utilized today.

\section{A. Object Representation}

- In a following, an article can be characterized as whatever is of intrigue. For instance, pontoons on the ocean, fish inside an aquarium, vehicles on a street, planes in the air.

- People strolling out and about are a lot of articles that might be critical to follow in a particular area. The appearance and shapes can be spoken to by object. First, we will portray the portrayal of article shape.

- Representation of articles is significant in object identification and following. There are different ways used to speak to objects.

- Points: The figures to the correct show the utilization of focuses in object portrayal.

- Primitive Geometric Shapes: Shapes like square shapes, ovals can be utilized to speak to objects.

- Object Silhouette and Contour: Contour portrayal characterizes the limit of an item. The area inside the form is known as the outline of the article.

\section{Difficulties And Problems in OBject Detection}

- Illumination: The lightning conditions may vary over the span of the day. Likewise, the climate conditions may influence the lighting in a picture

- Positioning: The adjustment in position must not influence the acknowledgment framework.

- Rotation: The picture can be in turned structure. The framework must be fit to deal with such trouble.

- Reflecting: The reflected picture of any article must be perceived by the item acknowledgment framework.

- Impediment: The condition when object in a picture isn't totally noticeable is alluded as impediment.

- Scale: Changes in the size must not influence the acknowledgment framework

\section{TeChniques FOR OBJect ReCOGNIZATION}

- Format Matching: Format coordinating is a strategy for discovering little pieces of a picture which coordinate a layout picture. It is a direct procedure. - Shading Based: The item 
discovery utilizing hues engaged with the articles is likewise essentially utilized and give an easy to execute technique. It gives powerful data to question acknowledgment. Shading histograms end up being basic and proficient and give an edge to the equivalent. The utilization and significance of shading traits for distinguishing objects has been proposed to us by Fahad Khan. This data has been sectioned into two methodologies which is the part based methodology and the proficient sub-window approach. Highlight blend, photometric invariance and smallness are the three significant highlights that should be considered while coordinating or attaching the shading traits with the article recognition. $\bullet$ Shape Based: Lately, shape has end up being critical in object acknowledgment. They have been investigated drastically to perceive questions in true familiar pictures. These highlights additionally give an advantage over nearby highlights like SIFT as the vast majority of the items are shown and portrayed by their shapes and surfaces, for example, various creatures and other shifting articles. They are no doubt used to add a bit of leeway to the nearby highlights. title or heads unless they are unavoidable.

\section{EXTRACTION OF OBJECTS}

Foundation Subtraction: The foundation deduction technique is the regular strategy for movement identification. It is an innovation that utilizes the distinction of the present picture and the foundation picture to distinguish the movement area, and is commonly ready to give information remembered for object data. The foundation picture is deducted from the present edge. On the off chance that the pixel distinction is more prominent than the set edge esteem $\mathrm{T}$, at that point it confirms that the pixels from the moving article, in any case, as the background pixels.

Background Subtraction: The aftereffect of picture groupings registered by the strategy here is in the accompanying figures. - When there is no movement in the frames: When there is no movement in the picture groupings then the contrast between the two pictures shows a dark double yield picture appears there is no distinction in a solitary pixel. $\bullet$ When there is movement in the edge: When there is movement in the scenes then the twofold picture of the distinction between the two edges shows movement having white shading and where there is no change shows dark shading.

\section{A. Applications}

1.Biometric recognition

2.Surveillance

3.Industrial inspection

4.Content - based image retrieval (CBIR)

5.Robotics

6.Medical analysis
7.Lane Detection

8. Vehicle Driver Assistance Systems.

9. Automated Surveillance.

10. Military Applications.

11. Security.

\section{IMPLEMENTATION}

In a video sequence an object is said to be in motion, if it is changing its location with respect to its background - The motion tracking is actually the process of keeping tracks of that moving object in video sequence i.e. position of moving object at certain time etc.

Method 1: Absolute Differences = Image subtraction $\mathrm{D}(\mathrm{t})=\mathrm{I}(\mathrm{ti})-\mathrm{I}(\mathrm{tj})$ Gives an image frame with changed and unchanged regions ged regions

Ideal Case for no motion: $\mathrm{I}(\mathrm{ti})=\mathrm{I}(\mathrm{t} \mathrm{j}), \mathrm{D}(\mathrm{t})=0$

\section{A. Absolute Difference}

Methods for Motion Detection Frame Differencing And Background Subtraction

\section{B. Drawbacks}

involves a lot of computations Not feasible for DSP implementation

\section{Figures and Tables}

\section{Conclusion}

In this introduction, we have diagrammed the accompanying focuses -

- Fundamental idea of Object Detection and Tracking. • Issues and challenges in Object Recognition. - Portrayal of articles. - Strategies in object acknowledgment. - Various and single article identification and AI process. - Item following. - Applications.

Therefore, we finish up - Object discovery is an errand of extricating Objects from explicit edges/pictures. - Object identification is one of the most broadly utilized idea in the field of Artificial Intelligence. - Has an incredible breadth in future for the advancement of the cutting-edge world.

\section{REFERENCES}

1. Himani Parekh, Darshan Thakore, UdesangJaliya, "A Survey On Object Detection And Tracking Methods", International Journal of Innovative Research in Computer and Communication Engineering, February 2014.

2. Kinjal Josh, Darshak Thakore"A Survey of Moving Object Detection and Tracking In Video Surveillance Systems", International Journal of Soft Computing And Engineering, July 2012.

3. Sukriti Srivastava, Ritika Singal, Manisha Lumb, "Efficient Lane Detection Algorithm using Different 
Filtering Techniques", International Journal of Computer Applications, February 2014

4. K. Suganya Devi, N. Malmurugan, M. Manikandan, "Object Motion Detection in Video Frames Using Background Frame Matching", International Journal of Computer Trends and Technology, vol.4, no. 6, pp. 1928-1931, June 2013.

5. Nan Lu, Jihong Wang, Q.H Wu, Li Yang, “An Improved Motion Detection Method for Real-Time Surveillance", Inter- national Journal of Computer Science, vol. 35, no. 1, Feb 2008.

6. Kinjal Josh, Darshak Thakore"A Survey of Moving Object Detection and Tracking In Video Surveillance Systems", International Journal of Soft Computing And Engineering, July 2012. 